\title{
Stretchable, Patch-Type, Wireless, 6-axis Inertial Measurement Unit for Mobile Health Monitoring
}

\author{
Jae Keun Lee, Kangil Kim, Sangmin Lee* \\ Department of Biomedical Engineering, Kyung Hee University, Yongin, Korea \\ Received 16 May 2019; received in revised form 08 July 2019; accepted 09 September 2019 \\ DOI: https://doi.org/10.46604/peti.2020.4272
}

\begin{abstract}
Wearable devices which measure and transfer signals from the human body can provide useful biometric data for various biomedical applications. In this paper, we present an implementation of the advanced Inertial Measurement Unit (IMU) with wireless communication technology for mobile health monitoring. The device consists of rigid silicon-based components on a flexible/stretchable substrate for applications in epidermal electronic devices to collect precise data from the human body. Using the Bluetooth Low Energy (BLE) System-on-a-chip (SoC), the device can be miniaturized and portable, and the collected data can be processed with low power consumption. The dimensions of the implemented system are approximately $40 \mathrm{~mm} \times 40 \mathrm{~mm} \times 100 \mu \mathrm{m}$. Also, the device can be attached closely to human skin, which results in minimized signal distortion due to body movements or skin deformations. In order to achieve device flexibility and stretch ability, the interconnection wires are designed as serpentine-shaped structures on a stretchable substrate. The previously reported "cut-and-paste" method is utilized to fabricate the device that produces complex, twisty interconnections with thin metal sheets. The implemented patch-type, wireless, 6-axis IMU is expected to have potential in various applications, such as health monitoring, dependency care, and daily lifelogging.
\end{abstract}

Keywords: Bluetooth low energy (BLE), inertial measurement unit (IMU), epidermal electronics, serpentine-shaped interconnection

\section{Introduction}

Recently reported researches have shown that the highly-reliable wearable devices which provide continuous physiological biometric information can be used for applications in mobile health [1-3], physical training [4], prosthetics [5] and human-machine interfaces (HMI) [6-8]. Especially, skin-like wearable electronic modules have soft form-factors to achieve close contact on the human body, which results in the significantly improved performance, such as the signal-to-noise ratio [9]. From the inherent skin-like characteristics of flexible and stretchable substrates, recent developments focus on the implementation of highly-scalable and modularized epidermal electronic devices for biomedical applications. Most commercialized wearable devices are implemented on a rigid substrate consist of silicon-based integrated circuit (IC) components to provide incomparable performance for signal processing with low-power consumption [10-11]. However, there is a large mechanical mismatch in the interface between the rigid circuit-board based devices and elastic human skin, which may reduce the accuracy of the measurement data. Thus, accurate biometric information can be measured using a hybrid integration scheme, where stretchable interconnection wires are used for elastic bio-interfaces and rigid silicon-based electronic ICs are used for calculations, signal processing and data transmission.

In order to improve the fidelity between the system and the skin, the implementation of the robust interconnection part is a fundamental technology to develop the system-level based epidermal electronic devices. Several groups have reported the

* Corresponding author. E-mail address: sangmlee@khu.ac.kr 
use of semiconductor microfabrication processes for implementing the serpentine-structured metal interconnects. This is achieved by fabricating a metal interconnection wire with a serpentine structure on the substrate using a semiconductor process, and then attaching the rigid electronic sensor with the ICs. Due to the serpentine-structured metal interconnects, the stress applied on the wires during elongation can be minimized. Although, microfabrication facilities for semiconductors are well-known to achieve precise micro patterns and minimized footprint for highly-miniaturized system, it is highly-expensive and time-consuming processes.

In this paper, an epidermal, patch-type 6-axis inertial measurement unit (IMU) is presented for biomedical applications, such as gait analysis, musculoskeletal disorders, and monitoring daily routines [12]. The proposed system is implemented using the packaged silicon-based ICs soldered on the bonding pads, which the metal interconnect between the ICs have serpentine-structures. Also, the whole device is implemented on a soft and stretchable platform to be attached on any part of human skin with high conformity. Each functional block of the system is fully-modular and reconfigurable, and this system is configured by a commercialized 6-axis accelerometer, a Bluetooth low energy (BLE) system-on-a-chip (SoC), several passive components and a chip antenna to measure the continuous biometric information with ease. For implementation of the proposed device, a simple, cleanroom-free manufacturing method is used. The detailed design schematic, fabrication process and performance evaluations will be discussed in the following sections. As a result, the system and the proposed method in this paper can be extended to the development of an advanced, multi-sensing, biometric platforms.

\section{Implementation Process}

The implemented epidermal, patch-type 6-axis IMU on a soft substrate is shown in Fig. 1. Sensors and devices used to construct the proposed system include 6-axis inertial sensor (MPU6050, Invernesses, San Jose, CA, USA), BLE SoCs (nRF52832, Nordic Semiconductor ASA, Norway), and several passive components. The serpentine-structured copper (Cu) wires are used to mount and integrate rigid electronic elements, which allows it to have elongation properties of the system under diverse deformation. The whole system is encapsulated using the stretchable adhesive film (Tegaderm, 3M, MN, USA), which can be attached to the human body and protects the system from moisture and dust. The system is implemented based on the modular concept, allowing the functional building blocks to be reconfigurable depending on the applications. By maintaining the signal processing parts, additional functions can be integrated by adding multiple sensors, such as electrodes, strain gauges or temperature sensors.

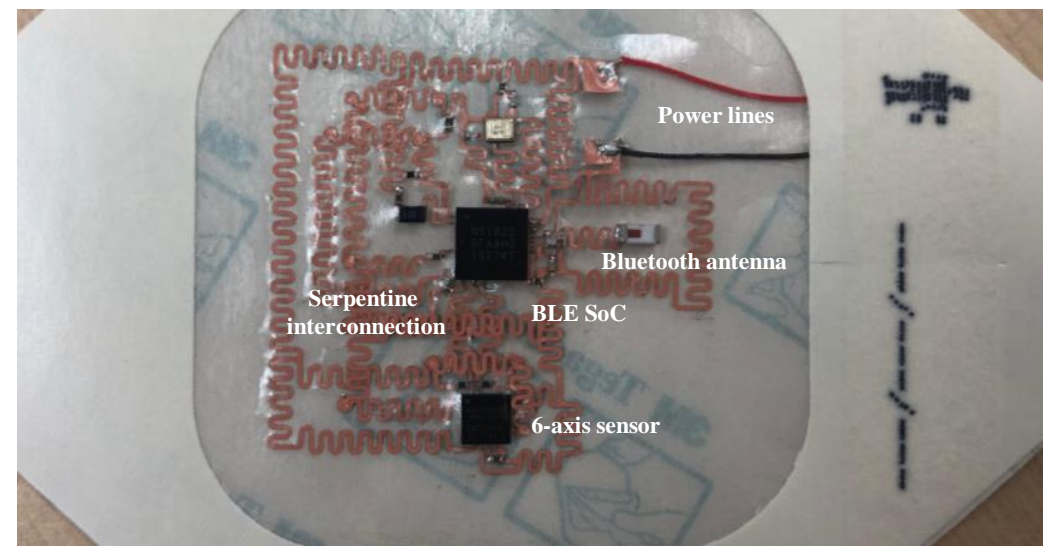

Fig. 1 Implemented epidermal, 6-axis IMU on stretchable platform

In Fig. 2, the block diagram of the epidermal, path-type 6-axis IMU is shown. The 6-axis inertial sensor and MCU transmit and receive data using the two-wire serial interface (TWI), which uses two bus signals (SCL and SDA) to exchange signals. The signals from the 6-axis inertial sensor are converted by the analog-to-digital converter (ADC), which is embedded in the BLE SoC, and the digitized data are spread by the universal synchronous/asynchronous receiver/transmitter (USART) structure in the signal conditioning unit. 


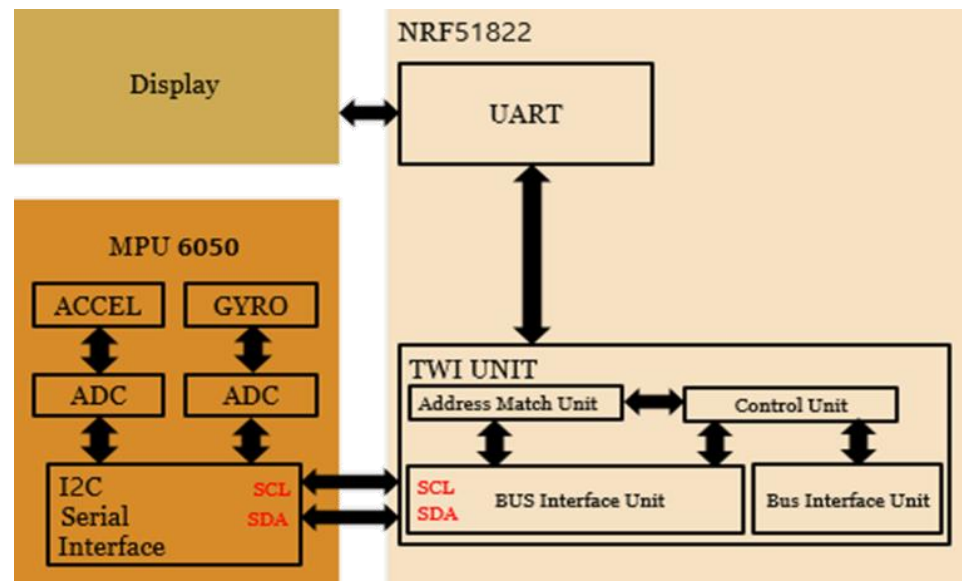

Fig. 2 Block diagram of the epidermal, 6-axis IMU using wireless data communication

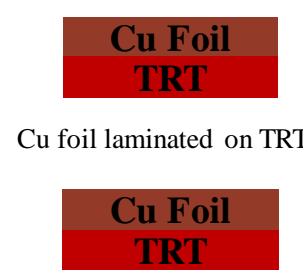

$\mathrm{Cu}$ foil patterning

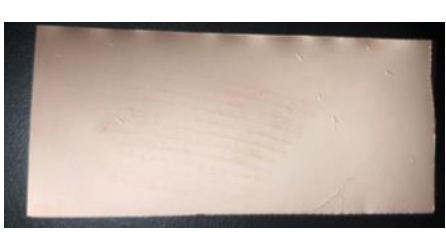

(a) Lamination

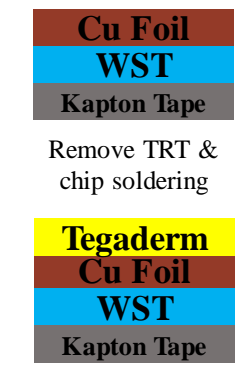

Laminate stretchable layer

(c) Soldering

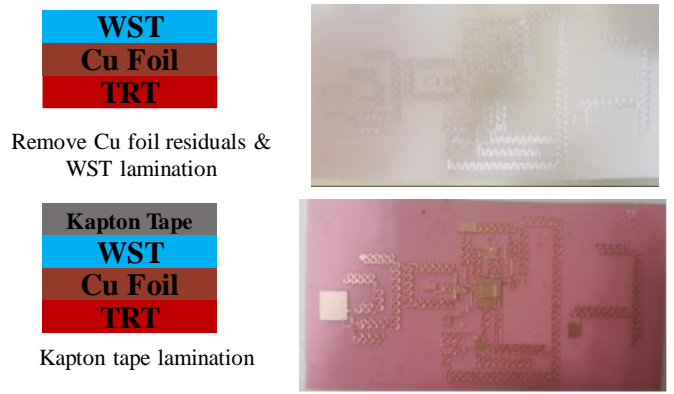

(b) Patterning

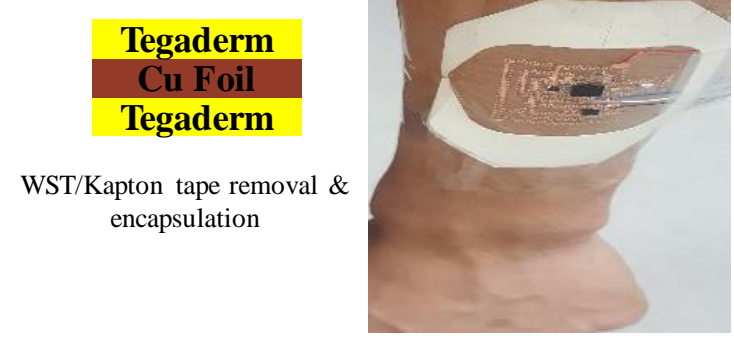

(d) Detachment and encapsulation

Fig. 3 Implementation process using "cut-and-paste" method

The implementation process to fabricate the patch-type 6-axis IMU on a flexible/stretchable polymeric surface is shown in Fig. 3. This process is used to design miniaturized systems on substrates with various form-factors using computer-aided design (CAD) program. To implement the proposed system, serpentine-structured interconnects and soldering pads for silicon IC assembly are designed. After designing the footprints of the system using CAD tool, a programmable cutter using sharp metal blade (Silhouette Cameo®, Silhouette America, USA) is equipped to define the interconnects and soldering pads. An 18- $\mu$ m-thick Cu foil (Copper 110 Annealed, Online Metals, USA) is laminated on the thermal release tape (TRT), and the Cu foil-laminated TRT is patterned according to the designed patterns programmed by the CAD tool. The blade depth of the cutting equipment is controlled only to cut the $\mathrm{Cu}$ foil layer. After cutting the $\mathrm{Cu}$ foil-laminated TRT, whole residual area except for designed patterns of $\mathrm{Cu}$ foil is removed. The patterned $\mathrm{Cu}$ foil laminated TRT layer is adhered with a Kapton film, water-soluble tape (WST) layer, which is bonded onto a glass substrate. And then, the whole bonded substrate is heated on a hot plate at $120{ }^{\circ} \mathrm{C}$ for 30 seconds to remove the TRT layer. After removing the TRT layer, a lead-free solder paste (ChipQuik, NY, USA) is used to assemble packaged ICs on the patterned $\mathrm{Cu}$ soldering pads. The electronic components, such as 6-axis inertial sensor, BLE SoC, chip antenna, passive components, and bridge interconnects are aligned on the soldering pads and heated on the hot plate at $230{ }^{\circ} \mathrm{C}$ for 3 minutes for fixation. Then, the soft adhesive film is covered on the circuit for encapsulation. The WST layer and the Kapton tape are removed to leave only electronic components and the 
serpentine-structured $\mathrm{Cu}$ interconnects. After covering the bottom layer of the device, the implementation of the patch-type 6 -axis IMU on a stretchable platform is finalized. The overall dimensions of the proposed system are approximately $40 \mathrm{~mm} \times$ $40 \mathrm{~mm}$, and the thickness is less than $100 \mu \mathrm{m}$ (excluding the ICs).

\section{Performance Evaluation}

The implementation results of the epidermal, 6-axis IMU are shown in Figs. (4)-(7), respectively. The stress applied to the interconnection wire during elongation can be minimized by its serpentine structure. Therefore, the modulus of the implemented device depends on the stiffness of the Tegaderm (approximately 7.4 MPa), which is similar to that of human skin (0.32 4 MPa). In Fig. 4, the connection to the wireless terminal using Bluetooth is shown. After the connection, short impact and tilting motions in a single direction are applied and measured wirelessly.

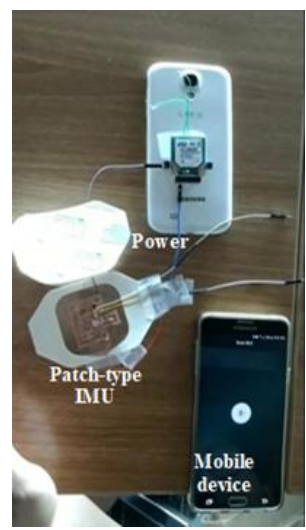

(a) Bluetooth connection

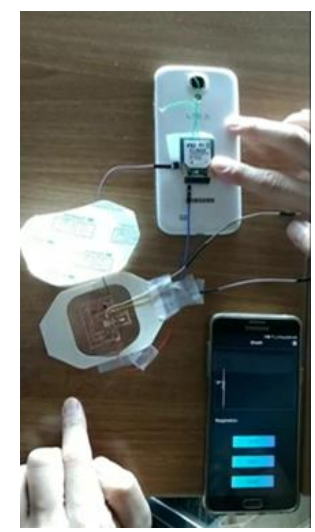

(b) With short impact

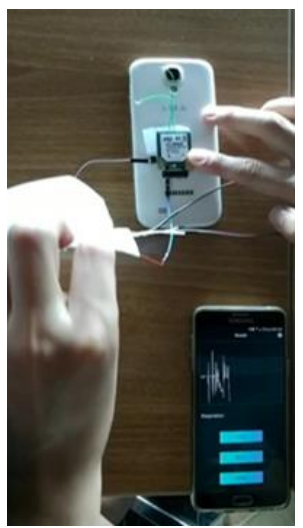

(c) Tilting in $\mathrm{x}$-axis

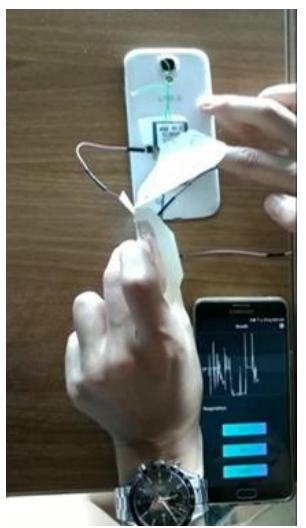

(d) Tilting in y-axis

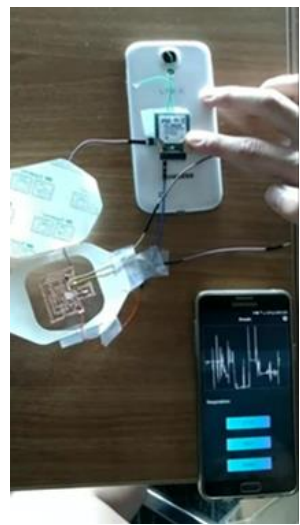

(e) Continuous no monitoring

Fig. 4 Wireless connection and continuous signal monitoring results
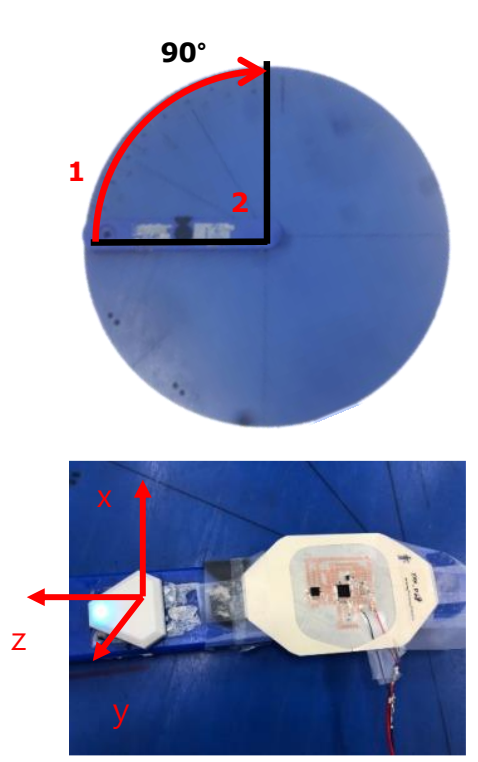
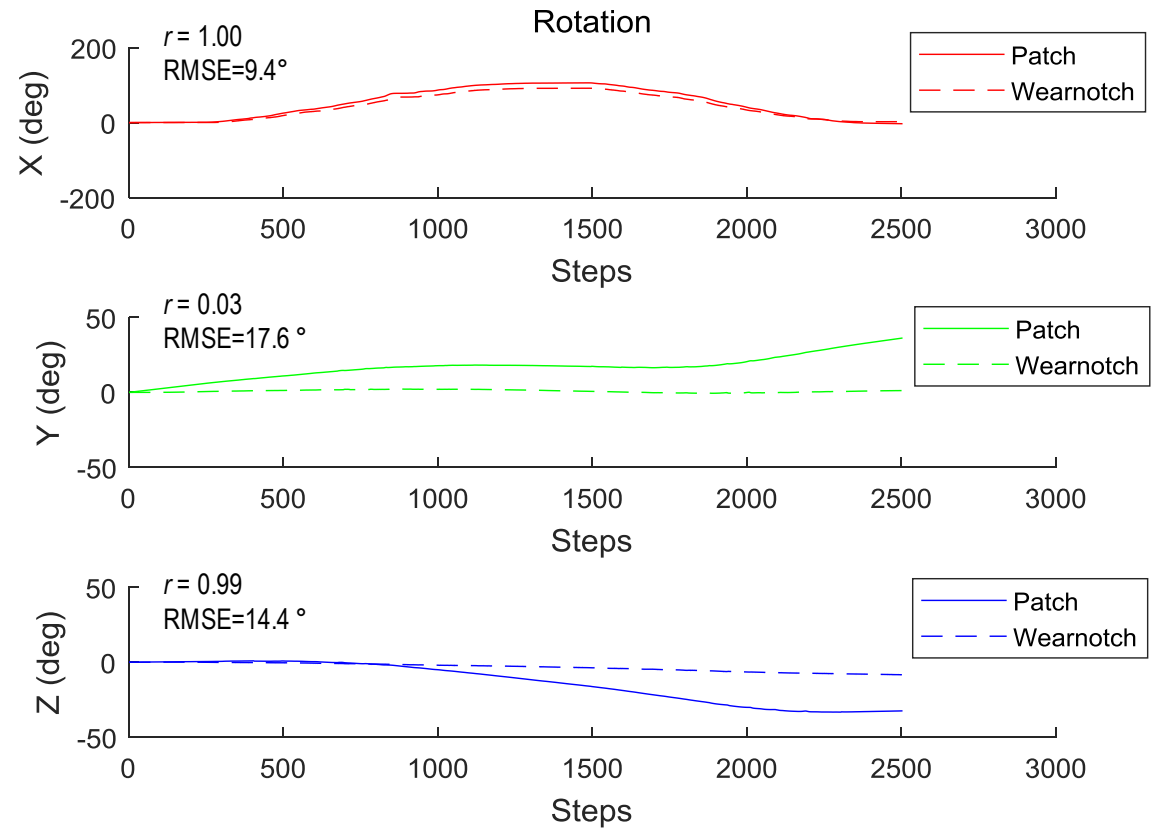

Fig. 5 Performance evaluation of the wireless, epidermal, 6-axis IMU for rotational motion

Also, experiments are conducted with applied rotational motion (Fig. 5) and arbitrary motion (Fig. 6) to the patch-type, 6-axis IMU attached to a human arm. In Fig. 5, the performance comparison results between the implemented patch-type device and Wear notch (Notch Interfaces, Inc., NY, USA) are shown. The maximum root-mean-square (RMS) error is about several tens of degrees. However, this appears to be an error due to the alignment mismatch and will be corrected through future calibration. The results show that real-time, continuous biometric motion data of the human body can be successfully 
monitored. In Fig. 7, the implemented patch-type, 6-axis IMU is attached to the arm to calculate the precise joint angle of the forearm. The flexibility and stretch ability of the implemented device allow it be utilized on the human body including the wrists, the ankles, the neck, and the abdominal region, which are common parts for monitoring the motion for dependency care and clinical diagnosis.

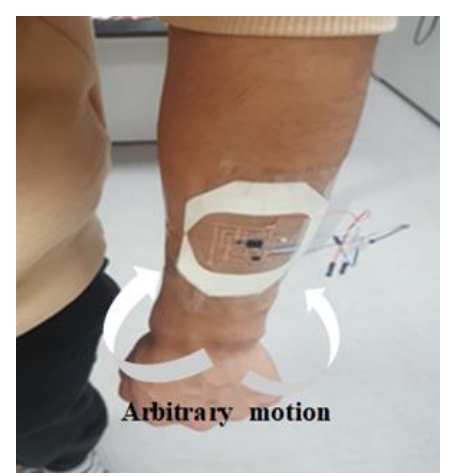

(a) Device attached on forearm

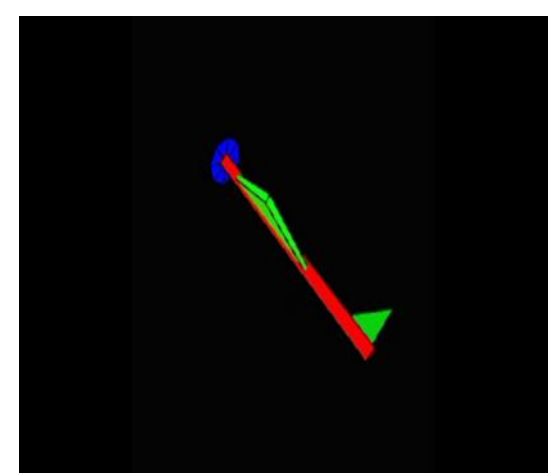

(b) Lateral translation

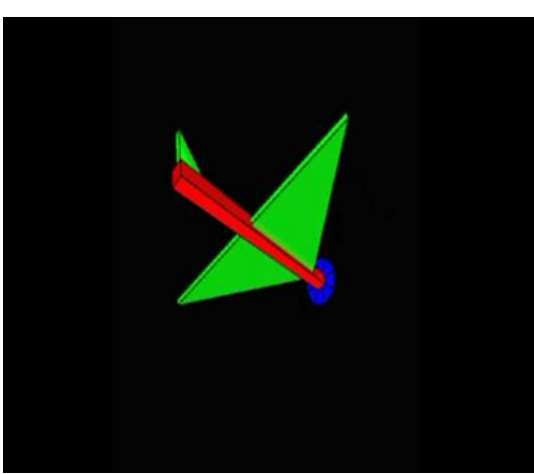

(c) Rotation

Fig. 6 Application result in 3-dimensional simulator control

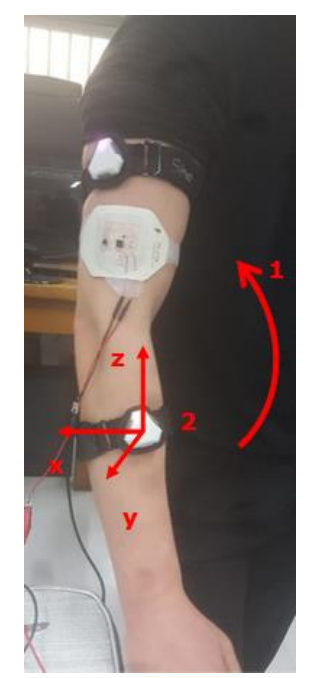

(a) Device attachment

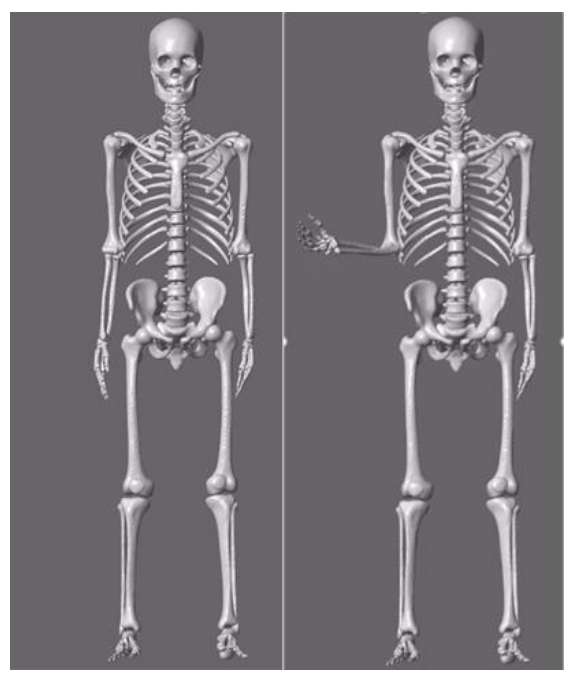

(b) Anterior view

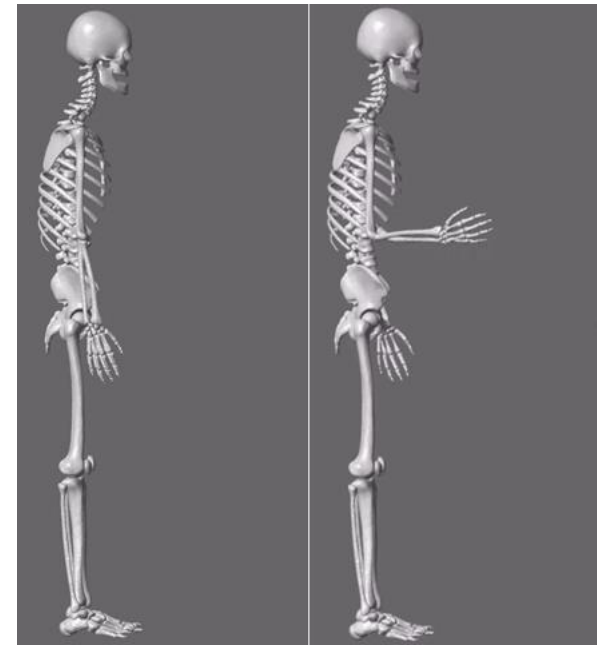

(c) Lateral view

Fig. 7 Joint angle analysis using the wireless, epidermal, 6-axis IMU

\section{Conclusions}

This paper presents an epidermal, patch-type 6-axis inertial measurement unit (IMU) for applications in wearable biomedical devices. The proposed device is implemented using the conventional circuit components on a soft adhesive substrate, and by firmly attached to the target, it is possible to collect accurate data from the human body. The planar dimensions of the implemented system are approximately $40 \mathrm{~mm} \times 40 \mathrm{~mm}$, and the overall thickness of the system, including the ICs, is less than $100 \mu \mathrm{m}$. A serpentine-structured interconnects are adopted between the circuit elements to achieve flexible and stretchable characteristics of the device. The preliminary implementation and experimental results of this paper demonstrated the potential for future applicability in various areas, such as dependency care and monitoring of human motion.

\section{Conflicts of Interest}

The authors declare no conflict of interest.

\section{Acknowledgments}

This work was supported by the National Research Foundation of Korea (NRF) grant funded by the Korea government (MSIP; Ministry of Science, ICT \& Future Planning (No. NRF-2017R1C1B5017561). This work was also supported by the 
National Research Foundation of Korea (NRF) grant funded by the Ministry of Science, ICT \& Future Planning for convergent research in human plus (No, NRF-2019078037).

\section{References}

[1] D. Kim, N. Lu, R. Ma, Y. Kim, R. Kim, S. Wang, et al., “Epidermal electronics,” Science, vol. 333, no. 6044, pp. 838-843, August 2011.

[2] H. Lee, T. K. Choi, Y. B. Lee, H. R. Cho, R. Ghaffari, L. Wang, et al., "A graphene-based electrochemical device with thermoresponsive microneedles for diabetes monitoring and therapy," Nature Nanotechnology, vol. 11, no. 6, pp. 566-572, 2016.

[3] A. Miyamoto, S. Lee, N. F. Cooray, S. Lee, M. Mori, N. Matsuhisa, et al., "Inflammation-free, gas-permeable, lightweight, stretchable on-skin electronics with nanomeshes," Nature Nanotechnology, vol. 12, no. 9, pp. 907-913, 2017.

[4] P. Salvo, A. Pingitore, A. Barbini, and F. Di Francesco, "A wearable sweat rate sensor to monitor the athletes' performance during training," Science \& Sports, vol. 33, no. 2, pp. e51-e58, October 2017.

[5] W. Gao, H. Ota, D. Kiriya, K. Takei, and A. Javey, "Flexible electronics toward wearable sensing," Accounts of Chemical Research, vol. 52, no. 3, pp. 523-533, 2019.

[6] Y. Liu, J. J. S. Norton, R. Qazi, Z. Zou, K. R. Ammann, H. Liu, et al., "Epidermal mechano-acoustic sensing electronics for cardiovascular diagnostics and human-machine interfaces," Science Advances, vol. 2, no. 11, pp. e1601185, November 2016.

[7] S. Huang, Y. Liu, Y. Zhao, Z. Ren, and C. F. Guo, "Flexible electronics: stretchable electrodes and their future," Advanced Functional Materials, vol. 29, no. 6, pp. 1805924, 2019.

[8] S. Lim, D. Son, J. Kim, Y. B. Lee, J. Song, S. Choi, et al., "Wearable electronics: transparent and stretchable interactive human machine interface based on patterned graphene heterostructures," Advanced Functional Materials, vol. 25, no. 3, pp. 375-383, November 2014.

[9] M. Kaltenbrunner, T. Sekitani, J. Reeder, T. Yokota, K. Kuribara, T. Tokuhara, et al., “An ultra-lightweight design for imperceptible plastic electronics," Nature, vol. 499, no. 7459, pp. 458-463, July 2013.

[10] Q. Cao, H. S. Kim, N. Pimparkar, J. P. Kulkarni, C. Wang, M. Shim, et al., "Medium-scale carbon nanotube thin-film integrated circuits on flexible plastic substrates," Nature, vol. 454, no. 7203, pp. 495-500, July 2008.

[11] B. Crone, A. Dodabalapur, Y. Y. Lin, R. Filas, Z. Bao, A. LaDuca, et al., "Large-scale complementary integrated circuits based on organic transistors," Nature, vol. 403, no. 6769, pp. 521-523, 2000.

[12] W. Teufl, M. Lorenz, M. Miezal, B. Taetz, M. Frohlich, and G. Bleser, "Towards inertial sensor based mobile gait analysis: event-detection and spatio-temporal parameters," Sensors, vol. 19, no. 1, pp. 38, 2019.

Copyright $\odot$ by the authors. Licensee TAETI, Taiwan. This article is an open access article distributed under the terms and conditions of the Creative Commons Attribution (CC BY-NC) license (https://creativecommons.org/licenses/by-nc/4.0/). 